\title{
Percepción de apoyo en duelo por profesionales y técnicos de cuidados intensivos pediátricos de hospitales públicos
}

\author{
Perception of support in professional's and technician's grief of pediatric \\ intensive care units in public hospitals
}

\author{
Vega Vega, Paula ${ }^{a}$, González Rodríguez, Rina ${ }^{a}$, López Encina, María Eugenia ${ }^{b}$, \\ Abarca González, Esmeralda ${ }^{a}$, Carrasco Aldunate, Paola ${ }^{a}$, \\ Rojo Suárez, Leticia ${ }^{c}$, González Briones, Ximena ${ }^{a}$
}

aEnfermera, Escuela de Enfermería, Pontificia Universidad Católica de Chile. Chile

bPsicooncóloga, Unidad de Oncología. Complejo Asistencial Dr. Sotero del Río. Chile

cPsicooncóloga, Unidad de Cuidados Paliativos, Complejo Asistencial Dr. Sotero del Río. Chile

Recibido: 7 de diciembre de 2018; Aprobado: 15 de abril de 2019

\begin{abstract}
Resumen
Introducción: La muerte en la infancia es una realidad a la que se ven enfrentados los profesionales de salud, especialmente en unidades de alta complejidad como son los cuidados intensivos. Esto genera en los profesionales impotencia y frustración, comprometiendo su salud física, emocional y mental, agravada por la sensación de bajo soporte social y deficiente preparación para el afrontamiento de la muerte y el apoyo a los familiares de sus pacientes. Objetivo: Develar la percepción de apoyo en duelo de profesionales y técnicos en unidades de cuidado intensivo pediátrico de hospitales públicos, tras la muerte de los pacientes. Sujetos y Método: Estudio cualitativo con enfoque fenomenológico. Se realizaron 16 entrevistas en profundidad a profesionales y técnicos de cuidados intensivos pediátricos de cinco hospitales públicos de Santiago. Como criterios de inclusión se consideraron el trabajar por más de un año en la unidad, haber vivenciado la muerte de pacientes, y reconocer que ha cursado un duelo profesional. La entrevista fue guiada por la siguiente pregunta: ¿Cómo ha vivenciado usted el apoyo en duelo recibido, tras la muerte de pacientes en su unidad? Una vez trascritas las narrativas se realizó el análisis fenomenológico y posterior triangulación de los datos, logrando la saturación. Resultados: Se develó que los participantes se sienten poco apoyados en sus duelos tras la muerte de los pacientes, existiendo obstáculos para su afrontamiento. Aunque enfrentar la muerte es algo complejo para ellos, reconocen que son capaces de generar estrategias de protección y además se sienten apoyados por personas de su entorno. A pesar de lo complejo de esta experiencia, ellos consideran que existen aprendizajes al enfrentar la muerte que los lleva a dar sentido a su labor profesional. Conclusión: Los profesionales requieren que las pérdidas en el ámbito laboral sean reconocidas, y por ello, contar con un apoyo formal y continuo de su equipo de trabajo e institución.
\end{abstract}

Palabras clave: Apoyo social; duelo; profesionales de salud; cuidados intensivos; niño 


\begin{abstract}
Introduction: Death in childhood is a reality faced by health professionals, especially in highly complex units such as intensive care ones. This leads to feelings of helplessness and frustration in health professionals, compromising their physical, emotional, and mental health, which is worsened by the feeling of low social support and poor preparation for coping with death and support for the patient's family. Objective: To expose the perception of sorrow support of professionals and technicians in pediatric intensive care units of public hospitals, after the death of the patients. Subjects and Method: Qualitative study with a phenomenological approach. 16 in-depth interviews were conducted with pediatric intensive care professionals and technicians from five public hospitals in Santiago, Chile. Inclusion criteria considered working for more than a year in the Unit, having experienced the death of patients, and recognizing that they have gone through a professional sorrow. The interview focused on the following question: How have you experienced the sorrow support received after the death of patients in your unit? Once the narratives were transcribed, the phenomenological analysis and subsequent data triangulation were carried out, achieving saturation. Results: It was observed that the participants feel little supported in their sorrows after the death of the patients, where there are obstacles to face the situation. Although facing death is something complex for the participants, they recognize that they can generate protection strategies and also feel supported by the people around them. Despite the complexity of this experience, the participants consider that there are lessons learned in facing death which leads them to give meaning to their professional work. Conclusion: Professionals need the recognition of deaths in the workplace and, therefore, formal and continuous support from their work team and institution.
\end{abstract}

\section{Keywords:}

Sorrow;

Social Support;

Health professionals;

Intensive care;

Child

\section{Introducción}

Aunque la medicina moderna se ha centrado en las últimas décadas en la prolongación de la vida y la curación de enfermedades, la muerte es una realidad que debe enfrentar a diario, en especial en unidades de alta complejidad $^{1,2}$. Para los equipos de unidades pediátricas, el fallecimiento de un niño se ha transformado en algo difícil de abordar, comprender y aceptar ${ }^{3,4}$. Por ello, este tipo de situaciones puede generar en los profesionales sensaciones de impotencia y frustración, lo que puede comprometer su estado físico, mental, emocional e incluso espiritual, llevándolos con el tiempo a un desgaste profesional ${ }^{5,6}$. Lo anterior, se puede ver agravado si el profesional se siente aislado y con bajo soporte social ante la muerte de los pacientes, sumado a la deficiente formación en pregrado para el afrontamiento de la muerte y apoyo a familiares ${ }^{7}$. Varias investigaciones han demostrado que el soporte social puede ser un mediador para enfrentar la muerte de los pacientes, ayudando a aliviar el estrés y permitiendo mejorar el estilo de afrontamiento frente a estas situaciones y así, favorecer el ambiente laboral en servicios de alta complejidad ${ }^{7,8}$. Sin embargo, este apoyo social está centrado en el reconocimiento de los vínculos entre pares, más que considerar la muerte de un paciente como una pérdida profesional y personal, existiendo así una diferencia entre el apoyo esperado y el recibido, desde su entorno como de la institución hospitalaria ${ }^{7,9}$. Por lo anterior, el objetivo de la presente investigación es develar la percepción de apoyado en duelo en profesionales de unidades de cuidado intensivo pediátrico (UCIP) de 5 hospitales de Santiago de Chile, tras la muerte de los pacientes.

\section{Sujetos y Método}

El presente estudio se realizó a través del diseño cualitativo, con enfoque fenomenológico según Husserl $^{9,10}$, lo que permite develar el verdadero significado de la vivencia humana respecto de un fenómeno, de la forma más original posible ${ }^{10,11}$. A través de un método reflexivo y subjetivo, que se desarrolla por medio de una metodología rigurosa, crítica y sistemática.

La muestra fue intencionada, invitando a participar a profesionales universitarios y técnicos que trabajan en unidades de cuidados intensivos pediátricos de cinco hospitales públicos de Santiago de Chile, entre los meses de mayo y septiembre del 2017.

Entre los criterios de inclusión que se consideraron fueron; trabajar por más de un año en la unidad, haber vivenciado la muerte de pacientes, reconocer que ha cursado un duelo profesional y que voluntariamente quisiera participar. Se excluyeron los funcionarios con duelo personal reciente, por recomendación de expertos. Los profesionales fueron invitados por email, y aquellos que aceptaron participar, fueron contactados por los investigadores para concertar la entrevista y tomar el consentimiento. 
La técnica de generación y análisis de datos se realizó según las etapas postuladas por Streubert ${ }^{11}$, iniciándose con la escritura del bracketing por parte de cada uno de los investigadores del proyecto. Los datos fueron recolectados a través de entrevistas en profundidad audio grabadas, las cuales fueron realizadas por 5 de las investigadoras capacitadas en la técnica (EA, PC, XG, ML y PV), usando un guion unificado. Cada entrevistadora se reunió con uno de los participantes en un lugar privado, fuera del hospital. Tras la lectura y firma del consentimiento informado se realizó la entrevista, guiada por la siguiente pregunta: ¿Cómo ha vivenciado usted el apoyo en duelo recibido, tras la muerte de pacientes en su unidad? Los audios fueron transcritos literalmente, y luego las narrativas se normalizaron por uno de los investigadores (PV). Éstas, una vez transcritas, fueron sometidas a un análisis comprensivo por las siete investigadoras en forma separada, quienes posteriormente realizaron la triangulación de sus hallazgos, llegando al consenso para develar las unidades de significado, agrupándolas en unidades mayores y así estructurar el fenómeno una vez saturados los datos. Los hallazgos fueron devueltos a los participantes, quienes expresaron sentirse representados. Durante el proceso se veló por el cumplimiento del rigor metodológico propuesto por Guba \& Lincoln ${ }^{10}$. Además, este estudio contó con la aprobación del Comité Ético Científico, MEDUC ( $\left.\mathrm{N}^{\circ} 16-329\right)$ y con el financiamiento del Fondo Nacional de Investigación en Salud (FONIS- SA16I0189).

\section{Resultados}

En el presente estudio participaron 16 profesionales de la salud, quienes a través de sus narrativas compartieron sus vivencias. Las características sociodemográficas de los participantes se muestran en la tabla 1.

\section{Hallazgos}

Tras el fallecimiento de los pacientes, los profesionales y técnicos enfrentan situaciones complejas que los llevan a sentirse emocionalmente comprometidos, vivenciando un duelo profesional. Sin embargo, el apoyo frente a esta situación lo perciben como bajo, generando la necesidad de visualizar esta temática y poder expresar su pesar en instancias protegidas. A pesar de lo anterior, estas vivencias traen consigo aprendizajes que les permite dar sentido a su labor profesional (figura 1).

A continuación, se analizará cada una de las unidades de significado develadas.

\section{Baja percepción de apoyo en duelo}

Los profesionales que laboran en UCIP perciben un bajo apoyo en duelo tras la muerte de sus pacientes,
Tabla 1. Características sociodemográficas de los participantes

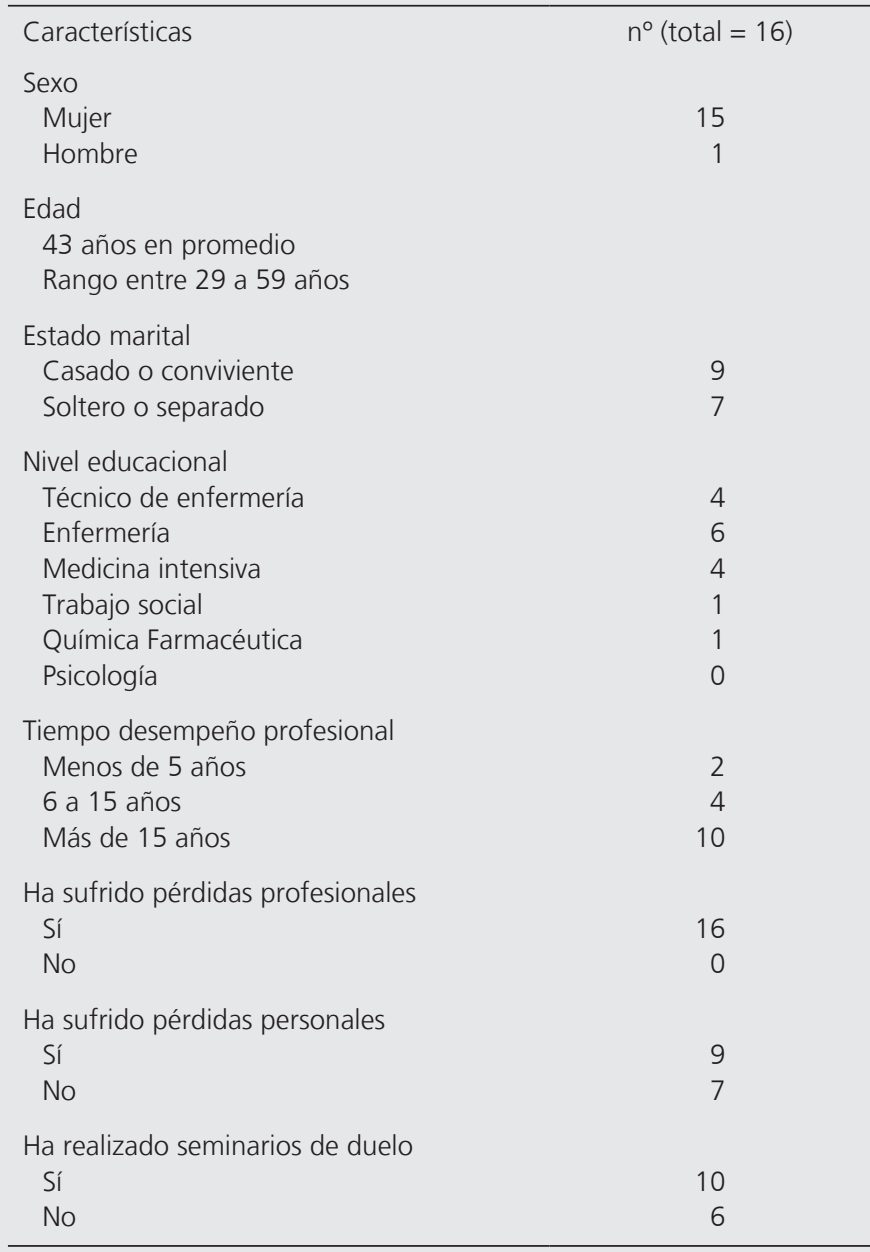

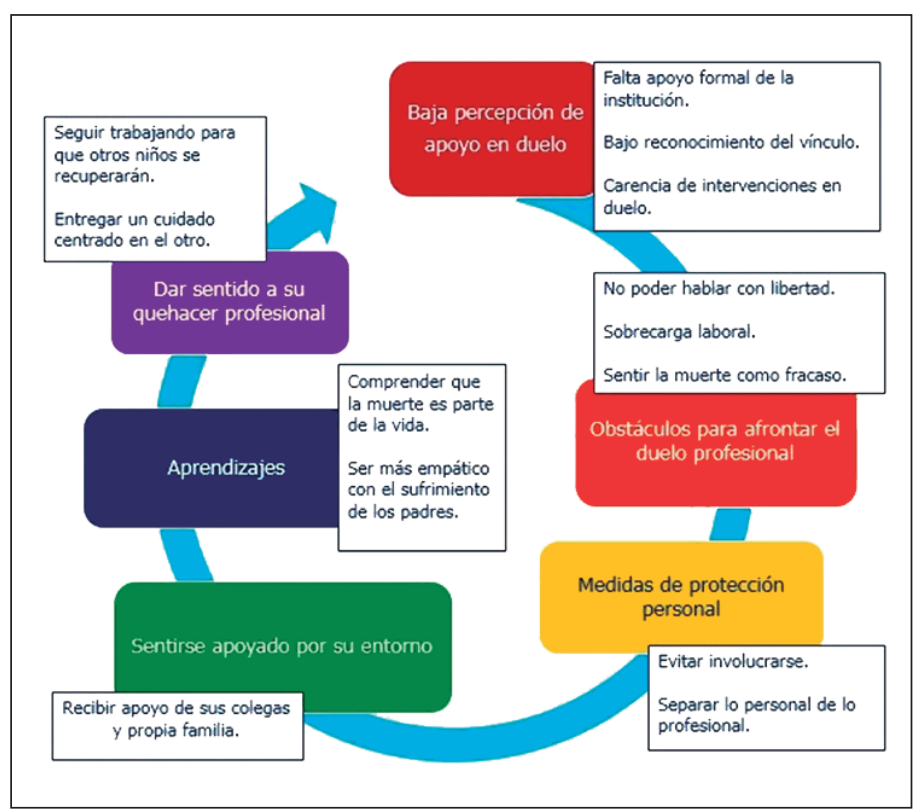

Figura 1. Percepción de apoyo en duelo en profesionales de UCIP. 
debido a la falta de un apoyo formal desde la institución, un bajo reconocimiento del vínculo y carencia de intervenciones en duelo hacia los equipos.

"La verdad que no es tema, ¡No! Yo nunca he escuchado, por ejemplo, de la Dirección o de la subdirección de enfermería que eso este dentro de alguna meta o alguna estrategia o como objetivo de salud mental del personal, relacionado a la pérdida de los pacientes... ¡Considerando que además somos un hospital pediátrico! Pero no, la verdad que no, yo no lo veo como que para ellos sea relevante. Nunca se ha tocado el tema, tampoco."

Enfermera, 38 años

"El problema es que yo no sé si con el tiempo la gente se vuelve dura. ¡Claro!... afecta de repente. A uno se le cae una lágrima; pero es como que dijeran: "es uno más". Entonces, cuando uno dice "ioh, qué pena se murió!, yo lo quería tanto, me dio mucha pena, lo siento por su mamá...” Mis compañeras me dicen: “¡Ay! ¡Qué te preocupas niña! Con los años se te va a pasar”.

Técnica paramédica, 52 años

"Si, el ambiente donde yo trabajo no es malo. Tenemos un buen trato entre enfermeras, médicos y nosotras, pero en ese aspecto falta esa intervención. Porque fallece el paciente, es triste y pena, pero hay que seguir y se limpia la unidad y listo, llega otro paciente y se olvidó lo que pasó."

Técnica paramédica, 45 años

\section{Obstáculos para afrontar el duelo profesional}

Los participantes sienten que existen obstáculos dentro de su quehacer profesional que no les permite expresar ni vivenciar adecuadamente su duelo, entre los que se develan; No poder hablar con libertad de las pérdidas, la sobrecarga laboral y sentir la muerte como fracaso.

"Pero también, como bien restringido. Porque en realidad no todas las personas están dispuestas a escucharte de cómo fue el proceso... qué sientes con la pérdida. No todos están vinculados como a la temática. Entonces, también se hace un poco complejo y ahi he tenido que ir regulando decir algunas cosas."

Trabajador social, 36 años

“... como es una UCI, hay que ayudar al otro niño. “YY! entonces, vamos por otro". Y es como un proceso... todo es como un proceso rápido que no da lugar para vivenciar el momento de la muerte del niño."

Químico farmacéutico, 40 años
"Ahora, obviamente la gente cuando... cuando le pone mucho empeño a que un paciente... viva y no muera, y finalmente el paciente muere, se siente derrotada, un fracaso, un fracaso tremendo."

Médica, 40 años

\section{Medidas de protección frente a las pérdidas}

La muerte de pacientes en la UCIP y el bajo apoyo percibido por los profesionales, les obliga a elaborar estrategias para protegerse, como es el evitar involucrarse y separar lo personal de lo profesional. Según lo relatado, estas medidas les permiten seguir trabajando en una unidad de alta complejidad, con alta carga emocional.

"Yo creo que las enfermeras, sobre todo las enfermeras más antiguas del hospital, es como que tienen "más lejanía”. O sea, la relación con los pacientes... “No!, hasta ahí nosotras, es un paciente. Tú lo atiendes, haces bien tu pega y todo lo que puedas hacer. ¡Pero hasta ahí llega!”. No se forma un vínculo como más allá."

Enfermera, 29 años

“ ¿Uno no se tiene que llevar el trabajo para la casa! Trato de no mezclar. Porque cada uno tiene su propio proceso. O sea, ¡cómo vas a sobrecargar más con lo que a ti te pasa al resto de la familia! Entonces... es un trabajo pesado, no es un trabajo fácil. "

Médica, 33 años

\section{Sentirse apoyado por su entorno}

A pesar de las vivencias de un apoyo deficiente frente a sus pérdidas profesionales, los participantes señalan que existe un apoyo informal y espontáneo de parte de las personas de su entorno, como son sus colegas y su propia familia. En este último caso, el apoyo lo reciben mayoritariamente de sus parejas.

"En el trabajo, recibo apoyo de mis colegas, que de alguna $u$ otra manera tuvieron que manejar al mismo paciente y de la jefa. Y en mi casa, de mi esposo. De hecho, a veces me siento medio culpable porque muchas veces le traspaso a él mucha de la angustia que yo puedo manejar en un turno. Pero en determinadas situaciones termino estresándolo a él también, porque voy soltando cosas y voy contando y el pobre termina también estresado. Sin embargo, mi esposo cuando me escucha y me abraza... ¡Para mí es súper importante el contacto físico desde el punto de vista afectivo!"

Médica, 35 años

"Por parte de los compañeros, sí siento apoyo. Sobre todo, por los que ya han sufrido pérdida de pacientes. Por 
los que son más nuevos... al contrario. Es uno, que lleva más tiempo, el que tiene que ir a apoyar al nuevo. Porque también para ellos es super difícil que se te muera un paciente en el turno... Por eso, apoyarse en equipo más que nada... y por el equipo de enfermeras o de técnicos, o con los médicos que uno tiene más cercanía... ¡Eso ayuda! "

Enfermera, 32 años

\section{Aprendizajes tras percibirse apoyado}

El percibirse apoyado al enfrentar las pérdidas al interior de los equipos, les ha permitido adquirir aprendizajes que los llevan a comprender que la muerte es parte de la vida y ser más empático con el sufrimiento de los padres de los pacientes.

"Me duelen igual... eso que dicen que uno se acostumbra. ¡Mentira! Yo creo que sí uno se pone barreras, o cosas, que unos dicen "tú aprendes a manejarlo y todo". A lo mejor en el sentido de que ahora sé que un paciente enfermo no siempre se va a ir a su casa... Que un paciente que llegó poli traumatizado, un quemado, no siempre va a volver a su casa. En eso sí... que yo creo que crecí... Porque antes pensaba que yo llegaba aquí y que todos los niñitos se iban a ir para su casa, que la muerte estaba como lejos, no la sentía cerca... ipero no es así! Pero eso yo ya, lo aprendí. “

Técnica paramédica, 38 años

"Aquí tenian visitas dos horas los padres. Entonces ha habido un cambio [ampliar el horario], que yo creo que nos ha hecho entender esta mirada de que cuerpo y mente no están separados. Que tenemos que acompañar a los papas en todo este proceso doloroso porque no es solamente el duelo. O sea, el ingresar a una unidad de cuidados intensivos es para los padres iimaginate!, que no se han separado nunca y más encima yo les digo: "no se puede quedar". Porque no tengo donde se queden..."

Médica, 56 años

“... poder ayudar, poder entregar algo a esa familia que está sufriendo. Y eso es como lo que aún, por lo menos a mí, me motiva como a seguir en esto. De hecho, por lo mismo entré a estudiar enfermería. O sea, mi tema era poder ayudar de una parte más humanizada que a lo mejor lo que puede hacer un médico. El poder entregar apoyo. Si al final está bien, podemos hacer muchas cosas, hay muchos procedimientos, hay muchas cosas y podemos aprender mucho. Sí. Pero el apoyo que entrega la enfermera es como fundamental en estos procesos de crisis... y con los niños con mayor razón."

Enfermera, 29 años

\section{Dar sentido a su quehacer profesional}

Los aprendizajes que la pérdida ha traído y la posibilidad de sentir apoyo de su entorno, les ha permitido dar sentido a su labor profesional, lo que los ha llevado a seguir trabajando para que otros niños se recuperen y entregar un cuidado centrado en el otro.

“... están graves y después uno los ve que salieron para arriba y tiraron, jtiraron para arriba y están vivos! A uno le da alegría eso... Ver a los pacientes que se mejoraron y están en su casa, después vienen a vernos acá. $Y .$. eso a uno como que lo reconforta. ¡Si!"

Técnica paramédica, 59 años

"Fijate que yo me motivo más por la tarea bien hecha, más que por el reconocimiento. Me ha tocado que los papas se acercan y lo agradecen... Recuerdo que yo voy pasando y al paciente lo habian bajado para que falleciera en el intermedio... Estaba en la última sala con un biombo. La mama lloraba sola.... Entonces como que en ese momento como que nadie se había detenido a ver que estaba pasando con ella. Yo justo pasé y la vi... Más que verla, sentí que sollozaba y entré. Le traje una silla, la hice sentarse, le pregunté si quería tomar a su hijo en brazos, que era un niñito que tenía algo hepático. Entonces me dice: “¿y lo puedo tomar?” ... "Pero por supuesto -le digo yo- si es su hijo”. “¿Conversaron con usted? ¿Usted está informada de todo...?" "Sí, señorita”, me dijo. Entonces yo la senté, tomé al niño, se lo pasé en brazos... Era lo que yo podía hacer. Entonces le digo yo, "iestá en condiciones usted de tomarlo?" "Sí señorita -me dijo- yo lo quiero tomar" se lo pasé, y me fui. Entonces me di un par de vueltas y ya después el chiquitito ya había fallecido..."

Enfermera, 35 años

\section{Discusión}

Tras este estudio, se pudo develar que los profesionales que trabajan en unidades de cuidados intensivos pediátricos vivencian duelos, al experimentar sentimientos de pesar y pérdida tras la muerte de un paciente. Para ellos es inevitable generar ciertos vínculos con los pacientes y/o su familia, por lo que esto impacta y repercute directamente en el cuidado y atención pre y post mortem que ellos brindan ${ }^{12}$. Así mismo, esta experiencia les exige ciertas habilidades de acompañamiento y apoyo a los familiares, para lo cual muchas veces no se sienten preparados ${ }^{12,13}$. En algunos casos, los profesionales consideran que deben ejecutarse acciones de preparación post mortem, sin atender a las propias necesidades emocionales del profesional, con una incongruencia entre el no sentirse listo para el duelo y el actuar ${ }^{14}$. 
En relación a lo anterior, los participantes relatan que el apoyo percibido frente a sus duelos en el ámbito profesional es deficiente, determinado por limitaciones en cuanto a falta de apoyo institucional, baja capacitación, sobrecarga laboral, el escaso tiempo para poder hacer cierres y ver la muerte como un fracaso, lo que está descrito en una serie de investigaciones realizadas en unidades de alta complejidad ${ }^{12-14}$. Para Kapoor et al. esta situación la describen como la necesidad de realizar una "pausa sagrada" entre los trabajadores de UCI, cuya falta genera en ellos un "cúmulo" de sufrimiento, afectando tanto su vida laboral como personal ${ }^{15}$. Según Peterson et al., esto estaría agravado por la falta de instancias para conversar dentro de los equipos en un ambiente protegido, como es la implementación de programas de afrontamiento al interior de las unidades $^{13}$. En cuanto a ver la muerte como fracaso, tanto los participantes como en los relatos de profesionales en otras investigaciones, develan que se sienten responsables frente a las muertes, al percibirse como los causantes directos o indirectos del fallecimiento del menor, generado en ellos emociones como la ira, impotencia, tristeza, desesperación, negación, sentimientos de vacío y vulnerabilidad ${ }^{15,16}$.

Por otra parte, narran que con el tiempo van generando estrategias individuales y grupales para superar la pérdida de los pacientes, como es el aceptar el apoyo dado por pares y sus propios familiares ${ }^{12,14}$. En cuanto a los pares, este apoyo se materializa en la posibilidad de compartir las vivencias de pérdida a través de conversaciones informales entre un grupo pequeño de funcionarios y/o la posibilidad de expresar libremente sus emociones en un ambiente protegido ${ }^{15,17,18}$. En el caso de su familia, Gálvez et al. señala que es dentro de ésta donde los profesionales se sienten mayormente contenidos, al encontrar comprensión y apoyo emocional ${ }^{14}$.

Sin embargo, varios profesionales a través de los años van desarrollando medidas de protección frente a las muertes, al mantenerse a distancia de los pacientes con el fin de no generar relaciones significativas ${ }^{13}$. Los expertos señalan que esta estrategia constituye un intento de apartarse del dolor y sufrimiento vivido, evitando pensar en ello y generando un distanciamiento emotivo, y así no caer en el riesgo de ser ineficientes en el actuar laboral, por culpa de sus emociones ${ }^{12,19}$. Esto también es compartido por los participantes del presente estudio.

A pesar de lo descrito anteriormente, en varios estudios los profesionales que trabajan en este tipo de unidades señalan que, a pesar de ser una situación compleja, el enfrentar la muerte de los niños y poder acompañar a las familias, les ha permitido ver la muerte como un proceso natural y cotidiano en el contexto laboral y con ello, desarrollar empatía frente al dolor de los padres ${ }^{9,14,16,20}$. Son estas vivencias, las que les han permitido darse cuenta que a pesar que los pacientes fallecen, el equipo hizo lo mejor que pudo haber hecho en ese momento ${ }^{14,18,20,21}$. Esta percepción también es compartida por los participantes, quienes lo ven como un aprendizaje del proceso de duelo, lo que les permite dar sentido a su quehacer y continuar trabajando en la unidad.

Tras el desarrollo del presente estudio, se considera como una limitante, la baja participación de hombres, a pesar de que la muestra fue intencionada para favorecer la participación equitativa y voluntaria de todos los funcionarios, independiente de su género. Esta diferencia en la participación entre hombres y mujeres se ha observado en otros estudios en profesionales de la salud, donde la participación masculina no supera el $20 \%$ 5,8,22,23. Cabe destacar que, en un estudio realizado en la misma población de la presente investigación, de los 210 participantes, solo el $11 \%$ eran hombres ${ }^{23}$. A su vez, los resultados mostraron que los hombres se perciben menos reconocidos en sus duelos que las mujeres ( $\mathrm{p}$ value $=0,039)$, lo cual abre la interrogante en cuanto al interés y necesidad que tienen los profesionales de salud que pertenecen al género masculino, de enfrentar las temáticas relacionadas con la muerte. Por lo que se sugiere, considerar esta población en futuras investigaciones que aborden este fenómeno desde la perspectiva cualitativa.

\section{Conclusión}

Los profesionales de unidades de UCI pediátricos requieren que las muertes de sus pacientes sean reconocidas como pérdidas profesionales, para lo que se considera fundamental recibir el apoyo de sus compañeros, familiares y de la propia institución donde trabajan. A pesar que existen obstáculos que no les permiten despedirse y dar un cierre a la relación con los pacientes ${ }^{15,16}$, ellos consideran que se podrían desarrollar ritos que favorezcan la elaboración de procesos de cierre a través de una pausa corta de tiempo dentro de su rutina laboral, considerándolas como "pausas sagradas"17. A eso se suma, el crear instancias para poder conversar de su pesar y de las propias vivencias ${ }^{13}$, pudiendo mejorar las instancias de comunicación dentro de equipo, y favorecer la cohesión entre compañeros, elemento clave en este tipo de procesos $^{24}$.

Según Kapoor et al. ${ }^{17}$, el desarrollar rituales de cierre, fomenta el trabajo en equipo, la apreciación de los esfuerzos y también, otorga un momento para dar sentido a lo entregado, reconociendo la gratitud y placer que significa dar cuidado a pacientes en la UCI.

Además, como se presentan en otras investigacio- 
nes ${ }^{7,12,13,14}$, los hallazgos de este estudio develan la necesidad concreta que tienen los profesionales de unidades intensivas de recibir apoyo formal por parte de las instituciones, a través de intervenciones de acompañamiento o capacitaciones en la temática de la muerte ${ }^{14,15}$, que les permitan reflexionar sobre sus aprensiones y miedos ante esta situación, y promover la búsqueda de significado de las experiencias ${ }^{20,21,25}$.

Tras lo analizado, toma relevancia el trabajar los duelos al interior de los equipos y en forma personal, puesto que permite dar sentido a la labor profesional, facilitando la generación y entrega de un cuidado humanizado al paciente y sus familias, lo que puede llegar a ser una estrategia de auto cuidado del profesional ${ }^{26}$.

Como todo estudio cualitativo, esta investigación permite la aproximación a la realidad entre los profesionales que comparten la vivencia del mismo fenómeno, y su posible transferencia de los datos develados sin llegar a generalizarlos. Se presenta entonces, el gran desafío de continuar la investigación en los procesos de duelo de los profesionales, abordando los distintos factores que influyen en el complejo fenómeno de afrontamiento de la muerte de pacientes, y en especial, los pacientes pediátricos.

\section{Responsabilidades Éticas}

Protección de personas y animales: Los autores declaran que los procedimientos seguidos se conformaron a las normas éticas del comité de experimentación humana responsable y de acuerdo con la Asociación Médica Mundial y la Declaración de Helsinki.

Confidencialidad de los datos: Los autores declaran que han seguido los protocolos de su centro de trabajo sobre la publicación de datos de pacientes.

Derecho a la privacidad y consentimiento informado: Los autores han obtenido el consentimiento informado de los pacientes y/o sujetos referidos en el artículo. Este documento obra en poder del autor de correspondencia.

\section{Conflicto de intereses}

Los autores declaran no tener conflicto de intereses.

\section{Financiamiento}

Esta investigación fue financiada por CONICYT, a través del Fonda Nacional de Salud. Proyecto FONIS SA $16 \mathrm{I} 0189$.

\section{Referencias}

1. Coca C, Arranaz P, Diéz-Asper H. Burnout en el personal sanitario que atienten a niños al final de su vida y a sus familiares. In: Gómez Sancho M, editor. Cuidados paliativos en el niño. España: Lerko/ GAFOS; 2007:167-88.

2. Flores R. Salud, Enfermedad y Muerte: Lecturas desde la Antropología Sociocultural. Revista MAD Universidad de Chile. 2004 [Internet] May; 10. Disponible en:http://www.rchdt. uchile.cl/index.php/RMAD/article/ viewFile/14783/15122.

3. Maza M, Zavala M, Merino J. Actitudes del profesional de enfermería ante la muerte de pacientes. Cienf. 2008; 15(1):39-48.

4. Hernández M. Duelo y Muerte: Una Confrontación Durante la Práctica de la Enfermería. Desarrollo Cienf Enferm 2008;16(2): 80-3.

5. Plante J, Cyr C. Health care professionals grief after death of child. Paediatr Child Health. 2011;16(4):213-6.

6. Olvera R. Téllez C. González A. Prevalencia de Burnout en trabajadores de un centro de salud. ATEN FAM. 2015; 22(2):46-9.

7. Anderson K, Ewen H, Miles E. The Grief support in Healthcare Scale. Nur Res. 2010;59(6):372-9.
8. Vega P, Bustos J González R, et al. Validación de escala de apoyo en duelo en atención de salud para población hispanoparlante. PSIC. 2015;12(2-3):35566.

9. Naidoo V, Sibiya MN. Experiences of Critical Care Nurses of Death and Dying in an Intensive Care Unit: A Phenomenological Study. J Nurs Care. 2014; 3(4): 179-183. DOI: https://doi. org/10.4172/2167-1168.1000179.

10. Husserl E. Ideas relativas a la fenomenología pura (3a ed.). México: Fondo Cultural de Economía. 1947.

11. Streubert H, Carpenter D. Qualitative research in nursing: advancing the humanistic imperative. (4a.ed). Philadelphia: Lippincott Williams \& Wilkins. 2007.

12. Donnelly S, Psirides A. Relatives' and staff's experience of patients dying in ICU. QJM An Int J Med [Internet]. 2015; 108(12):935-42. Disponible en: http:// dx.doi.org/10.1093/qjmed/hcv059

13. Peterson J, Johnson M, Halvorsen B, et al. What is it so stressful about caring for a dying patient? A qualitative study of nurses' experiences. Int J Palliat Nurs [Internet]. 2010;16(4):181-7. Available from: https:/doi.org/10.12968/ ijpn.2010.16.4.47784.

14. Gálvez M, Ríos F, Fernández L, del Águila B, Muñumel G, Fernández C. El final de la vida en la Unidad de Cuidados Intensivos desde la perspectiva enfermera: Un estudio fenomenológico. Enfermeria Intensiva. 2011;22(1):13-21. https://doi. org/10.1016/j.enfi.2010.11.003.

15. Rashotte J, Fothergill-Bourbonnais F, Chamberlain M. Pediatric intensive care nurses and their grief experiences: a phenomenological study. Heart \& Lung [Internet]. 1997;26(5):372-86. Disponible en: https://doi.org/10.1016/S0147-9563 (97)90024-8

16. Lee J, Dupree C. Staff Experiences with End-of-Life Care in the Pediatric Intensive Care Unit. JPM. 2008;11(7):986-90. https://doi.org/10.1089/jpm.2007.0283.

17. Kapoor S, Morgan CK, Siddique MA, Guntupalli KK. "Sacred Pause" in the ICU: Evaluation of a Ritual and Intervention to Lower Distress and Burnout. Am J Hosp Palliat Med [Internet]. 2018;(0). Disponible en: https://doi. org/10.1177/1049909118768247.

18. Attia A, Abd-Elaziz W, Kandeel N. Critical care nurses' perception of barriers and supportive behaviors in end-of-life care. Am J Hosp Palliat Med [Internet]. 2013; 30 (3):297-304. Disponible en: https://doi. org/10.1177/1049909112450067.

19. García V, Rivas E. Experiencia de enfermeras intensivistas pediátricas en la muerte de un niño: vivencias, duelo, 
aspectos bioéticos. Cienc. enferm. 2013; 19(2):111-24. https://doi.org/10.4067/ s0717-95532013000200011.

20. Lopera M. Accompanying in death is a pillar of care, a painful duty and a dilemma. Cienc. enferm. 2016;22(1):6574. https://doi.org/10.4067/S071795532016000100006.

21. Peterson JL, Johnson MA, Halvorsen $\mathrm{B}$, et al. Where do nurses go for help? A qualitative study of coping with death and dying. International Journal of Palliative Nursing. 2010;16(9):432-8. https://doi. org/10.12968/ijpn.2010.16.9.78636.

22. Anderson K, Ewen H, Miles E. The Grief support in Healthcare Scale. Nurs Res 2010;59:372-9. Doi: 10.1097/ NNR.0b013e3181fca9de.

23. Vega P, González R, Bustos J, et al. Relación entre apoyo en duelo y el síndrome de burnout en profesionales y técnicos de oncología y cuidados intensivos pediátricos. Rev Chilena de Ped. 2017;88(5):614-21.

24. Gouveia C, Oliver D. Can Addressing Death Anxiety Reduce Health Care
Workers' Burnout and Improve Patient Care? J Palliat Care. 2011;24(4):287.

25. Neimeyer R. Complicated grief and the reconstruction of meaning: conceptual and empirical contributions to a cognitive-constructivist model clinical. Psychology: science and practice. 2006;13(2):141-5. doi: 10.1111/j.14682850.2006.00016.x.

26. Watson J. Intentionality and CaringHealing Consciousness: A Practice of Transpersonal Nursing. Holistic Nursing Practice. 2002;16(4):12-9. 